\title{
Quantifying Service Compatibility: A Step beyond the Boolean Approaches
}

\author{
Meriem Ouederni ${ }^{1}$, Gwen Salaün ${ }^{2}$, and Ernesto Pimentel ${ }^{1}$ \\ 1 University of Málaga, Spain \\ \{meriem, ernesto\}@lcc.uma.es \\ 2 Grenoble, INP-INRIA-LIG, France \\ Gwen.Salaun@inria.fr
}

\begin{abstract}
Checking the compatibility of service interfaces allows one to avoid erroneous executions when composing services together. In this paper, we propose a flooding-based approach for measuring the compatibility degree of service interfaces specified using interaction protocols. This proposal is fully automated by a prototype tool we have implmented.
\end{abstract}

\section{Introduction}

Checking the compatibility of service interfaces guarantees the safe reuse and the successful interoperation of services in the context of Service Oriented Computing (SOC). In this paper, we focus on the interaction protocol level of service interfaces. Checking the compatibility of interaction protocols is a tedious and hard task even though this is of utmost importance to avoid run-time errors, e.g., deadlock situations or unmatched messages. Most of the existing approaches (see for instance [5172863]) return a "True" or "False" result to detect whether services are compatible or not. Unfortunately, a Boolean answer is not very helpful for many reasons. First, in real world case studies, there will seldom be a perfect match, and when service protocols are not compatible, it is useful to differentiate between services that are slightly incompatible and those that are totally incompatible. Furthermore, a Boolean result does not give a detailed measure of which parts of service protocols are compatible or not.

To overcome the aforementioned limits, a very few works (see for instance [16]) recently aim at measuring the compatibility degree of service interfaces. However, most of them are based upon description models of service interfaces which do not consider value-passing and internal behaviours ( $\tau$ transitions). Moreover, they often measure the interface compatibility using a simple (i.e., not iterative) traversal of protocols, and consider a unique compatibility notion making their application quite restricted.

In this paper, we propose a generic framework where the compatibility degree of service interfaces can be automatically measured according to different compatibility notions. We illustrate our approach using a bidirectional compatibility notion, namely unspecified receptions. Additional notions can easily be added to our framework. We consider a formal model for describing service interfaces with interaction protocols (messages and their application order, but 
also value-passing and internal actions). In our approach, the compatibility degree is computed in two steps. A first step computes a set of static compatibility degrees where the execution order of messages is not taken into account. Then, a flooding algorithm computes the compatibility degree of interaction protocols using the static compatibility results. The computation process also returns the mismatch list indicating the interoperability issues. The proposed framework is fully automated by a prototype tool (called Comparator) we have implemented.

The remainder of this paper is structured as follows. Section 2 describes our model of services. Section 3 introduces the compatibility notion we use in this paper for illustration purposes. In Section 4 , we present our solution for measuring the service compatibility. Section 5 introduces our prototype tool. Finally, concluding remarks are presented in Section 6. All the formal definitions and more details are given in a companion technical report available at Meriem Ouederni's Webpage.

\section{Service Model}

We assume service interfaces are described using their interaction protocols represented by Symbolic Transition Systems (STSs) which are Labelled Transition Systems extended with value-passing (parameters coming with messages). In particular, a STS is a tuple $(A, S, I, F, T)$ where: $A$ is an alphabet which corresponds to the set of labels associated to transitions, $S$ is a set of states, $I \in S$ is the initial state, $F \subseteq S$ is a nonempty set of final states, and $T \subseteq S \backslash F \times A \times S$ is the transition relation. In our model, a label is either an (internal) $\tau$ action or a tuple $(m, d, p l)$ where $m$ is the message name, $d$ stands for the communication direction (either an emission ! or a reception ?), and $p l$ is either a list of typed data terms if the label corresponds to an emission (output action), or a list of typed variables if the label is a reception (input action) 1

It is worth noticing that communication between services relies on a synchronous and binary communication model. The operational semantics of this model is given in 6]. STSs can also be easily derived from higher-level description languages such as Abstract BPEL, see for instance 7 14,4 where such abstractions were used for verification, composition or adaptation of Web services.

\section{Unspecified Receptions Compatibility}

Compatibility checking verifies the successful interaction between services wrt. a criterion set on their observable actions. This criterion is referred to as a compatibility notion. We distinguish two classes of notions depending on the direction of the compatibility checking, that are, bidirectional and unidirectional analysis. Our approach supports both classes; here we particularly illustrate it with a bidirectional compatibility notion, namely unspecified receptions ( $U R$ for short). This notion is inspired from [17] and requires that two services are compatible (i) if they are deadlock-free, and (ii) if one service can send a message at a reachable state, then its partner must eventually receive that emission such that both

\footnotetext{
${ }^{1}$ The message names and parameter types respect the service signature.
} 
services evolve into states where the $U R$ notion is preserved. In real-life cases, one service must receive all the requests from its partner, but can also accept more receptions, because the service could interoperate with other partners.

\section{Measuring Protocol Compatibility}

This section presents our techniques for measuring the compatibility of two service protocols. In what follows, we describe a transition using a tuple $\left(s, l, s^{\prime}\right)$ such that $s$ and $s^{\prime}$ denote the source and target states, respectively, and $l$ stands for its label. We suppose that for all transitions $\left(s, \tau, s^{\prime}\right), s \neq s^{\prime}$. Given two services described using STSs $S T S_{i \in\{1,2\}}=\left(A_{i}, S_{i}, I_{i}, F_{i}, T_{i}\right)$, we define a global state as a pair of states $\left(s_{1}, s_{2}\right) \in S_{1} \times S_{2}$. All the compatibility measures we present in the sequel belong to [0..1] where 1 means a perfect compatibility. The approach overviewed in Figure 1 consists first in computing a set of static compatibility measures (Section 4.1). In a second step, these static measures are used for computing the behavioural compatibility degree for all global state in $S_{1} \times S_{2}$ (Section 4.2). Last, the result is analysed and a global compatibility degree is returned (Section 4.3).

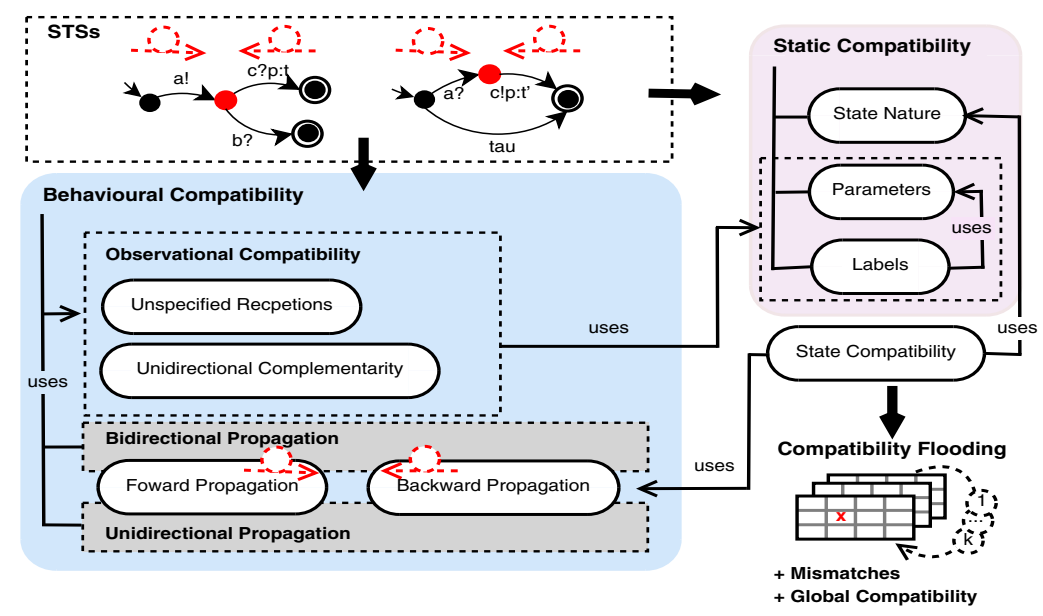

Fig. 1. Compatibility Measuring Process

\subsection{Static Compatibility}

We use three auxiliary static compatibility measures, namely state nature, labels, and exchanged parameters.

State Nature. The comparison of state nature assigns 1 to each pair of states which have the same nature, i.e., both states are initial, final or none of them. Otherwise, the measure is equal to 0.

Parameters. The compatibility degree of two parameter lists $p l_{1}$ and $p l_{2}$ depends on three auxiliary measures, namely: (i) the compatibility of parameter 
number comparing the list sizes; (ii) the compatibility of parameter order measuring the number of types which does not appear in the same order, and (iii) the compatibility of parameter type using the set of unshared types in both lists. These measures must be set to 1 if these lists are empty.

Labels. Protocol synchronisation requires that compatible labels must have opposite directions. Therefore, given a pair $\left(l_{1}, l_{2}\right) \in A_{1} \times A_{2}$, the label compatibility - lab-comp $\left(l_{1}, l_{2}\right)$ - is measured as 0 if these labels have same directions. Otherwise, the computation of this measure uses the semantic distance between message names and the parameter compatibility degree presented above. Here, message names are compared using the Wordnet similarity package [13].

\subsection{Behavioural Compatibility}

We consider a flooding algorithm which performs an iterative measuring of behavioural compatibility for every global state in $S_{1} \times S_{2}$. This algorithm incrementally propagates the compatibility between neighbouring states using backward and forward processing. The compatibility propagation is based on the intuition that two states are compatible if their backward and forward neighbouring states are compatible, where the backward and forward neighbours of global state $\left(s_{1}^{\prime}, s_{2}^{\prime}\right)$ in transition relations $T_{1}=\left\{\left(s_{1}, l_{1}, s_{1}^{\prime}\right),\left(s_{1}^{\prime}, l_{1}^{\prime}, s_{1}^{\prime \prime}\right)\right\}$ and $T_{2}=\left\{\left(s_{2}, l_{2}, s_{2}^{\prime}\right),\left(s_{2}^{\prime}, l_{2}^{\prime}, s_{2}^{\prime \prime}\right)\right\}$ are the states $\left(s_{1}, s_{2}\right)$ and $\left(s_{1}^{\prime \prime}, s_{2}^{\prime \prime}\right)$, respectively. The flooding algorithm returns a matrix denoted $C O M P_{C N, D}^{k}$ where each entry $C O M P_{C N, D}^{k}\left[s_{1}, s_{2}\right]$ stands for the compatibility measure of global state $\left(s_{1}, s_{2}\right)$ at the $k^{\text {th }}$ iteration. The parameter $C N$ refers to the considered compatibility notion which must be checked according to $D$, that is, a bidirectional $(\leftrightarrow)$ protocol analysis in this paper. $C O M P_{C N, D}^{O}$ represents the initial compatibility matrix where all states are supposed to be perfectly compatible, i.e., $\forall\left(s_{1}, s_{2}\right) \in S_{1} \times S_{2}, C O M P_{C N, D}^{0}\left[s_{1}, s_{2}\right]=1$. Then, in order to compute $C O M P_{C N, D}^{k}\left[s_{1}, s_{2}\right]$, we consider the observational compatibility function, $o b s-c o m p p_{C N, D}^{k}$, and the state compatibility function, state-comp $p_{C N, D}^{k}$, which combines the forward and backward propagations. In this paper, we only present the forward compatibility for lack of space, the backward compatibility can be handled in a similar way based upon incoming rather than outgoing transitions.

Unspecified Receptions. For all global state $\left(s_{1}, s_{2}\right)$ : (i) obs-comp $p_{U R, \leftrightarrow}^{k}$ returns 1 if and only if every outgoing emission at state $s_{1}$ (and $s_{2}$ ) perfectly matches an outgoing reception at state $s_{2}$ (and $s_{1}$ ) and all synchronisations on those emissions lead to compatible states; (ii) $o b s-c o m p_{U R, \leftrightarrow}^{k}$ returns 0 if there is a deadlock; (iii) otherwise, obs-comp $p_{U R, \leftrightarrow}^{k}$ measures the best compatibility of every outgoing emission at $s_{1}$ with the outgoing receptions at $s_{2}$, leading to the neighbouring states which have the highest compatibility degree, and vice-versa.

Example 1. Let us consider the global state $\left(\mathrm{s}_{0}, c_{0}\right)$ in Figure 2. Here, there is a unique emission seek! at $c_{0}$ which perfectly matches with search? at $s_{0}$, lab-comp $($ seek!, search? $)=1$ using Wordnet similarity package. The synchronisation on these compatible labels leads to $\left(s_{1}, c_{1}\right)$ where $C O M P_{U R, \leftrightarrow}^{O}\left[s_{1}, c_{1}\right]=1$. 


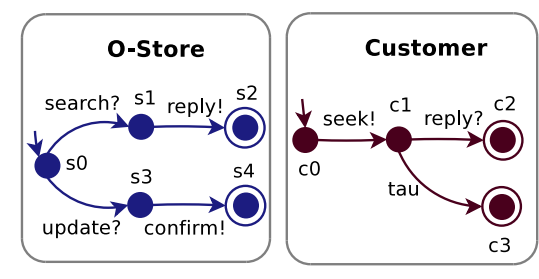

Fig. 2. Online Store

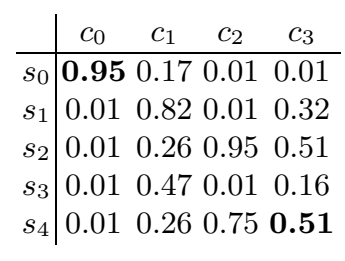

Fig. 3. Matrix $C O M P_{U R, \leftrightarrow}^{7}$

Thus, at the first iteration, obs-comp ${ }_{U R, \leftrightarrow}^{1}\left(\left(s_{0}, c_{0}\right)\right)=$ lab-comp $($ seek $!$, search? $) *$ $C O M P_{U R, \leftrightarrow}^{O}\left[s_{1}, c_{1}\right]=1$.

Forward Propagation. The compatibility is computed from both services point of view. The function $f w-$ propag $_{C N, \leftrightarrow}^{k}\left(\left(s_{1}, s_{2}\right)\right)$ propagates to $\left(s_{1}, s_{2}\right)$ the compatibility degrees obtained for the forward neighbours of state $s_{1}$ with those of state $s_{2}$, and vice-versa. For each $\tau$ transition, fw-propag $g_{C N, \leftrightarrow}^{k}$ must be checked on the target state. Observable transitions going out from $\left(s_{1}, s_{2}\right)$ are compared using obs-comp ${ }_{C N, \leftrightarrow}^{k}\left(\left(s_{1}, s_{2}\right)\right)$.

Example 2. Let us consider the global state $\left(s_{1}, c_{1}\right)$ in Figure 2. We show below the computation of fw-propag $g_{U R, \leftrightarrow}^{1}\left(\left(s_{1}, c_{1}\right)\right)$ which results in the average of the auxiliary values computed from each protocol point of view:

$f w-\operatorname{propag}_{U R, \leftrightarrow}^{1}\left(\left(s_{1}, c_{1}\right)\right)=\frac{1}{2} *$

where:

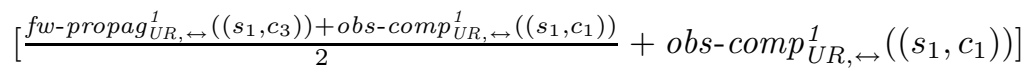

- fw-propag $\operatorname{UR}_{U, \leftrightarrow}^{1}\left(\left(s_{1}, c_{3}\right)\right)=$ obs-comp $_{U R, \leftrightarrow}^{1}\left(\left(s_{1}, c_{3}\right)\right)=0$ due to the deadlock that can occur at the state $\left(s_{1}, c_{3}\right)$.

- obs-comp ${ }_{U R, \leftrightarrow}^{1}\left(\left(s_{1}, c_{1}\right)\right)=$ lab-comp (reply?, reply! $) * C O M P_{U R, \leftrightarrow}^{O}\left[s_{2}, c_{2}\right]=1$.

As a consequence, fw-propag $g_{U R, \leftrightarrow}^{1}\left(\left(s_{0}, c_{0}\right)\right)=\frac{3}{4}$.

State Compatibility. The function state-comp ${ }_{C N, D}^{k}\left(\left(s_{1}, s_{2}\right)\right)$ computes the weighted average of three measures: the forward and backward compatibilities, and the value returned by the function comparing state natures.

Compatibility Flooding. As a final measuring step, $C O M P_{C N, D}^{k}\left[s_{1}, s_{2}\right]$ is computed as the average of its previous value $C O M P_{C N, D}^{k-1}\left[s_{1}, s_{2}\right]$ and the current state compatibility degree. Our iterative process terminates when the Euclidean difference $\varepsilon_{k}=\left\|C O M P_{C N, D}^{k}-C O M P_{C N, D}^{k-1}\right\|$ converges.

Example 3. Figure 3 shows the matrix computed for the example depicted in Figure 2. This matrix was obtained after 7 iterations. Let us comment the compatibility of states $c_{0}$ and $s_{0}$. The measure is quite high because both states are initial and the emission seek! at $c_{0}$ perfectly matches the reception search? at $s_{0}$. However, the compatibility degree is less than 1 due to the backward propagation of the deadlock from the global state $\left(s_{1}, c_{3}\right)$ to $\left(s_{1}, c_{1}\right)$, and then from $\left(s_{1}, c_{1}\right)$ to $\left(s_{0}, c_{0}\right)$. 
Mismatch Detection. Our compatibility measure comes with a list of mismatches which identifies the incompatibility sources, e.g., unmatched message names or unshared parameter types. For instance, the states $s_{0}$ and $c_{1}$ in Figure 2 present several mismatches, e.g., the first state is initial while the second is not, and their outgoing transition labels have the same directions.

Extensibility. Our approach is generic and can be easily extended to integrate other compatibility notions. Adding a compatibility notion $C N$ only requires to define a new function obs-comp ${ }_{C N, D}^{k}$.

\subsection{Analysis of Compatibility Measures}

Compatible Protocols. Our flooding algorithm ensures that every time a mismatch is detected in a reachable global state, its effect will be propagated to the initial states. Hence, the forward and backward compatibility propagation between neighbouring states implies that protocols are compatible if and only if their initial states are also compatible. Such information is useful for automatically discovering available services that can be composed without using any adaptor service for compensating mismatches [10].

Global Protocol Compatibility. As regard incompatible protocols, this global measure is helpful to differentiate between services that are slightly incompatible and those which are totally incompatible, and for service ranking and selection. Seeking for services with high global compatibility degree enables to simplify further processing to compensate existing mismatches, e.g., using service adaptation. In our approach we compute the global compatibility degree as the weighted average of all behavioural compatibility degrees that are higher than a threshold $t$. The weight is the rate of states having a compatibility degree higher than $t$, among all states compared in one service, with the states in the partner service.

\section{Prototype Tool}

Our approach for measuring the compatibility degree of service protocols has been fully implemented in a prototype tool called Comparator which has been

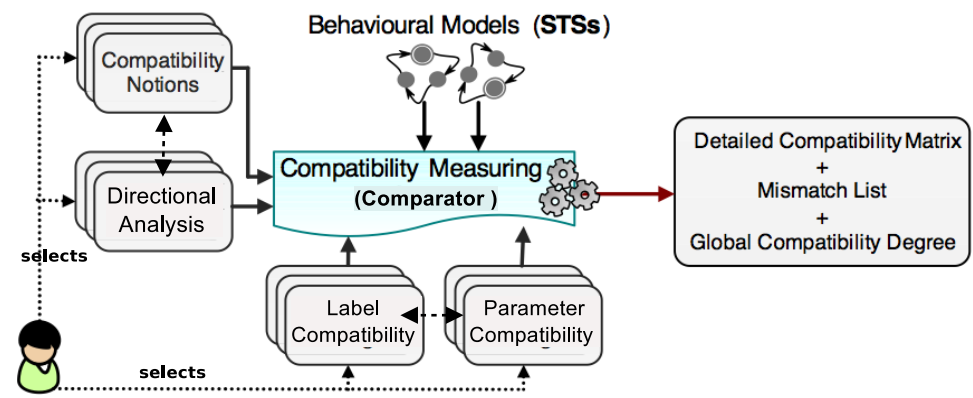

Fig. 4. Comparator Architecture 
validated on more than 110 examples. The framework architecture is given in Figure 4. The Comparator tool, implemented in Python, accepts as input two XML files corresponding to the service interfaces and an initial configuration, i.e., the compatibility notion, the checking direction, and a threshold $t$. The tool returns the compatibility matrix, the mismatch list, and the global compatibility degree which indicates how compatible both services are. The implementation of our proposal is highly modular which makes easy its extension with new compatibility notions, or other strategies for comparing message names and parameters.

\section{Concluding Remarks}

Existing quantitative analysis of service behaviours have been devoted to two related issues in SOC. A first research line measures the compatibility, that is, how much interacting services fulfill each other's requirements. The second research line checks the substitutability, that is, the correspondences between similar service behaviours. The techniques used for measuring the simulationbased correspondences in [15], and the global compatibility degree based on checking the deadlock-freedom in [16] of two protocols, rely on a simple (not iterative), forward, and parallel traversal. In 91], the authors compute the minimal edit distance between two similar versions of one service interface. Other works [1112 rely on a similarity flooding algorithm for computing the matrix of correspondences between models such as labelled graphs and statecharts.

Our work advances the state-of-the-art as follows. Our behavioural model takes value passing and internal behaviours into account. Our framework is generic, supports different compatibility notions, and can be extended with other ones. Considering both the forward and backward compatibility propagations makes our flooding algorithm more precise, and also enables to detect the boolean compatibility. Our proposal is fully supported by the Comparator tool which has been validated on many examples. The global compatibility degree is helpful for service selection and ranking. The returned matrix and the mismatch list have some straightforward applications in the software adaptation area [10 17. Our tool was already integrated into an environment for the interactive specification of adaptation contracts [4]. Our main perspective is to apply our compatibility measuring approach for the automatic generation of adaptor protocols.

Acknowledgements. This work has been partially supported by the project TIN2008-05932 funded by the Spanish Ministry of Innovation and Science and FEDER, and by the project P06-TIC220, funded by the Andalusian government.

\section{References}

1. Aït-Bachir, A.: Measuring Similarity of Service Interfaces. In: Bouguettaya, A., Krueger, I., Margaria, T. (eds.) ICSOC 2008. LNCS, vol. 5364. Springer, Heidelberg (2008) 
2. Bordeaux, L., Salaün, G., Berardi, D., Mecella, M.: When are Two Web Services Compatible?. In: Shan, M.-C., Dayal, U., Hsu, M. (eds.) TES 2004. LNCS, vol. 3324, pp. 15-28. Springer, Heidelberg (2005)

3. Bravetti, M., Zavattaro, G.: Contract-Based Discovery and Composition of Web Services. In: Bernardo, M., Padovani, L., Zavattaro, G. (eds.) SFM 2009. LNCS, vol. 5569, pp. 261-295. Springer, Heidelberg (2009)

4. Cámara, J., Salaün, G., Canal, C., Ouederni, M.: Interactive Specification and Verification of Behavioural Adaptation Contracts. In: Proc. of QSIC 2009, pp. 65-75. IEEE Computer Society, Los Alamitos (2009)

5. de Alfaro, L., Henzinger, T.: Interface Automata. In: Proc. of ESEC/FSE 2001, pp. 109-120. ACM Press, New York (2001)

6. Durán, F., Ouederni, M., Salaün, G.: Checking Protocol Compatibility using Maude. In: Proc. of FOCLASA 2009. ENTCS, vol. 255, pp. 65-81 (2009)

7. Fu, X., Bultan, T., Su, J.: Analysis of Interacting BPEL Web Services. In: Proc. of WWW 2004, pp. 621-630. ACM Press, New York (2004)

8. Hameurlain, N.: Flexible Behavioural Compatibility and Substitutability for Component Protocols: A Formal Specification. In: Proc. of SEFM 2007, pp. 391-400. IEEE Computer Society, Los Alamitos (2007)

9. Lohmann, N.: Correcting Deadlocking Service Choreographies Using a SimulationBased Graph Edit Distance. In: Dumas, M., Reichert, M., Shan, M.-C. (eds.) BPM 2008. LNCS, vol. 5240, pp. 132-147. Springer, Heidelberg (2008)

10. Mateescu, R., Poizat, P., Salaün, G.: Adaptation of Service Protocols Using Process Algebra and On-the-Fly Reduction Techniques. In: Bouguettaya, A., Krueger, I., Margaria, T. (eds.) ICSOC 2008. LNCS, vol. 5364, pp. 84-99. Springer, Heidelberg (2008)

11. Melnik, S., Garcia-Molina, H., Rahm, E.: Similarity Flooding: A Versatile Graph Matching Algorithm and Its Application to Schema Matching. In: Proc. of ICDE 2002, pp. 117-128. IEEE Computer Society, Los Alamitos (2002)

12. Nejati, S., Sabetzadeh, M., Chechik, M., Easterbrook, S.M., Zave, P.: Matching and Merging of Statecharts Specifications. In: Proc. of ICSE 2007, pp. 54-64. ACM Press, New York (2007)

13. Pedersen, T., Patwardhan, S., Michelizzi, J.: WordNet:Similarity - Measuring the Relatedness of Concepts. In: Proc. of AAAI 2004, pp. 1024-1025. AAAI, Menlo Park (2004)

14. Salaün, G., Bordeaux, L., Schaerf, M.: Describing and Reasoning on Web Services using Process Algebra. IJBPIM 1(2), 116-128 (2006)

15. Sokolsky, O., Kannan, S., Lee, I.: Simulation-Based Graph Similarity. In: Hermanns, H., Palsberg, J. (eds.) TACAS 2006. LNCS, vol. 3920, pp. 426-440. Springer, Heidelberg (2006)

16. Wu, Z., Deng, S., Li, Y., Wu, J.: Computing Compatibility in Dynamic Service Composition. Knowledge and Information Systems 19(1), 107-129 (2009)

17. Yellin, D.M., Strom, R.E.: Protocol Specifications and Component Adaptors. ACM Trans. Program. Lang. Syst. 19(2), 292-333 (1997) 